\title{
Relationship between Expansibility of Arterial Wall and Serum Triglyceride Levels in Treated Diabetics*
}

\author{
Hiroshi Kudo \\ The Second Department of Medicine (Prof. T. Kimura), \\ Iwate Medical University, Moriaka
}

\begin{abstract}
The pulse wave velocity from the origin of the aorta to the radial artery was measured in diabetics, in order to determine whether it is related to the fasting levels of serum triglycerides and total serum cholesterol.

A high degree of negative correlation was found between the expansibility of arterial wall and serum triglyceride levels in well-controlled diabetics as well as poorly controlled ones. There was also a similar correlation with total serum cholesterol levels, only in well-controlled diabetics.
\end{abstract}

In 1922, Bramwell and Hill ${ }^{1,2}$ established the following theoretical principle on the expansibility of arterial wall; the expansibility of arterial wall can be theoretically expressed as a function depending only on the pulse wave velocity in the artery. This is based on the physical fact that an increase in the hardness of an object causes an acceleration of the conduction velocity of the wave in the object. Thereafter, many clinical investigations as to the pluse wave velocity in hypertension or diabetes mellitus have been reported.

Since Kato et al. ${ }^{3 \sim 5}$ stressed that the expansibility of arterial wall had close relation to age, sex, blood pressure and serum cholesterol, the quantitative estimation of the degree of arteriosclerosis has been noticed as a reliable method for the clinical diagnosis of arteriosclerosis. However, no study has hitherto been reported on the relation between the expansibility of arterial wall and serum triglycerides.

The present author tried to correlate the expansibility of arterial wall with the levels of serum total cholesterol and serum triglycerides in diabetics.

\section{Materials and Methods}

All subjects investigated were in- and out-patients with established diagnosis of diabetes mellitus in Iwate Medical University Hospital, and for over one year they were treated with insulin or sulfonylurea in combination with dietary regulation.

They were classified into two groups according to the fasting blood sugar levels by Hagedorn-Jensen's method:

1) Well-controlled diabetics, whose fasting venous blood sugar levels were under

Received for publication, August 22, 1968.

* Clinical Studies of Serum Triglyceride Levels in Diabetes Mellitus, III.

The outlines of the studies described in this papar were presented at the 10th Annual Meeting of the Japan Diabetic Society, Nagoya, April, 1967. 
$130 \mathrm{mg} \%$ in over $80 \%$ of the measurements. They wore 12 males and 6 females with a mean age of 56 years.

2) Poorly controlled diabetics, whose fasting venons blood sugar levels were over $130 \mathrm{mg} \%$ under the above-said condition. They were 14 males and 5 females with a mean age of 49 years.

About ont hour before the pulse wave velocity was measured, blood samples were obtained from all patients in the fasting state. Subsequently, serum total cholesterol and serum triglycerides were measured; the former by a modification of Zuckerman and Natelson's ${ }^{6}$ or Ota's ${ }^{7}$ method, the latter by a modification of Van Handel and Zilversmit's method. ${ }^{8}$

The pulse wave velocity in the artery from the origin of the aorta to the radial artery was measured by the method of Kato et al.: ${ }^{3}$ Electrocardiogram and radial pulse wave were simultaneously recorded, and the length of the arsery from the origin of the aorta to the site of the ladial artery from which the pulse was recorded was determined according to the equation, $y=1.155 x+10$, where $y$ is the length of the artery in cm and $x$, the length of the arm in $\mathrm{cm}$ of the test subject. The measurements were made between 9 and 10 a.m., in the fasting state, at room temperature $\left(18-23^{\circ} \mathrm{C}\right)$, the subjects resting in the supine position.

The expansibility of arterial wall as calculated according to the following equation of Bramwell and Hill:1,2

$$
\text { Expansibility }=\frac{12.7}{(\text { Pulse wave velocity } \mathrm{m} / \mathrm{sec})^{2}}
$$

\section{Results}

Relationship between pulse wane velocity and serum total cholesterol (Fig. 1)

In well-controlled diabetics, there was a good positive correlation between the pulse wave velocity and the serum total cholesterol levels $(n=18, r=+0.60,0.01<$ $p<0.02)$. However, in poorly controlled diabetics no significant correlation was found $(n=18, r=+0.15,0.5<p<0.6)$.

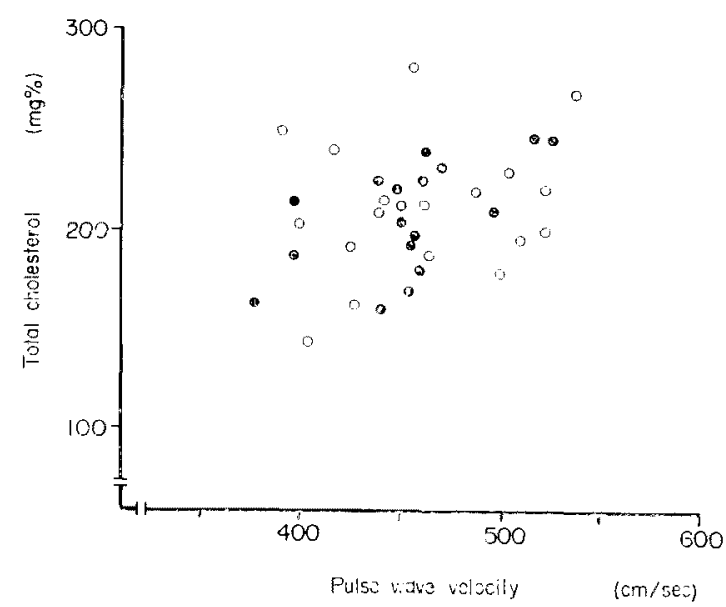

Fig. 1. Relationship between pulse wave velocity and serum total cholesterol levels in welland poorly controlled diabetics.

- Well-controlled diabetios $n=18 \quad r=+0.60(0.01<p<0.02)$

- Poorly controlled diabetics $n=18 \quad r=+0.15 \quad(0.5<p<0.6)$ 
Relationship between expansibility of arterial wall and serum total cholesterol (Fig. 2)

In well-controlled diabetics, a good negative correlation between the expansibility of arterial wall and the serum total cholesterol levels was found $(n=$ $18, r=-0.56,0.01<p<0.02)$. However, there was no significant correlation in poorly controlled diabetios $(n=18, r=-0.14,0.1<p<0.2)$.

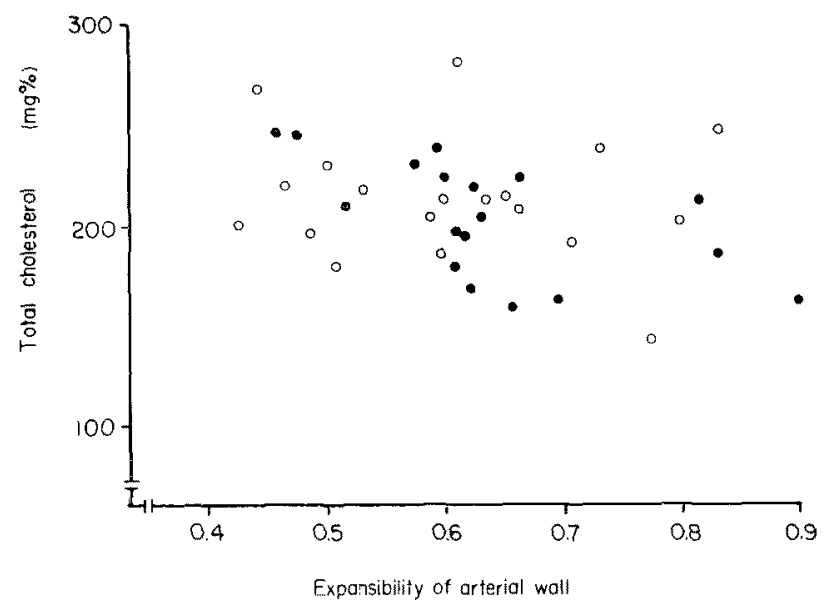

Fig. 2. Relationship between expansibility of arterial wall and serum total cholesterol levels in well and poorly controlled diabetics.

- Well-controlled diabetics $n=18 \quad r=-0.56(0.01<p<0.02)$

C. Poorly controlled diabetics $n=18 \quad r=-0.14(0.1<p<0.2)$

Relationship between pulse wave velocity and serum triglycerides (Fig. 3)

In well-controlled diabetics, there was a good positive correlation between the pulse wave velocity and the serum triglyceride levels $(n=18, r=+0.60, p<$ $0.01)$. There was also a good positive correlation in poorly controlled diabetics $(n=19, r=+0.50,0.02<p<0.05)$.

Relationship between expansibility of arterial wall and serum triglycerides (Fig. 4)

In well-controlled diabetics, there was a good negative correlation between the expansibility of arterial wall and the serum triglyceride levels $(n=18, r=-0.59$, $0.01<p<0.02$ ). Also, poorly controlled diabetics were found to display a good negative correlation $(n=19, \quad r=-0.57,0.01<p<0.02)$. The serum triglyceride levels tended to be higher in poorly controlled diabetics than in well-controlled ones.

\section{Discussion}

In 1960 , Kato et al. ${ }^{5}$ demonstrated that the expansibility of arterial wall had a good negative correlation with the total serum cholesterol levels in arterio- 


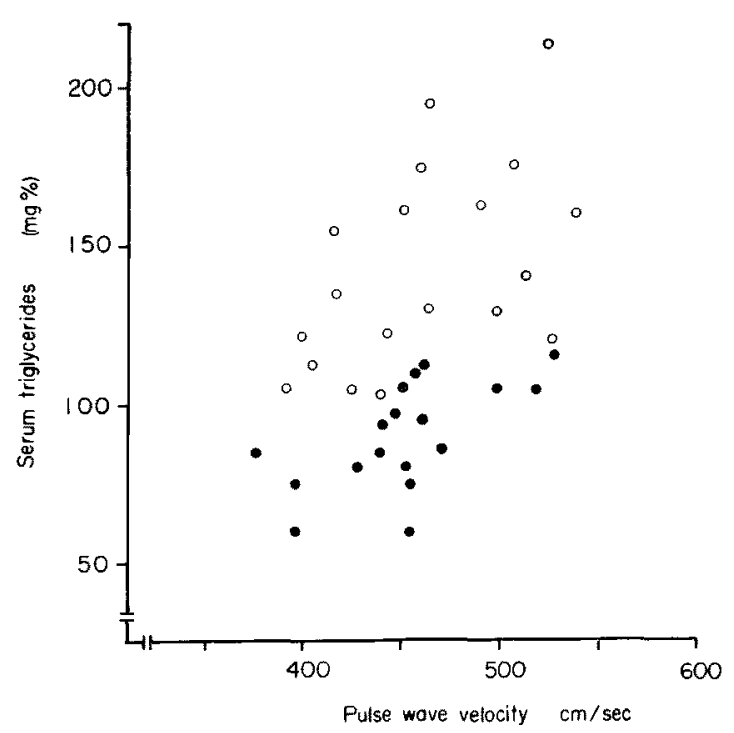

Fig. 3. Relationship between pulse wave velocity and serum triglyceride levels in well. and poorly controlled diabetics.

- Well-controlled diabetics $n=18 \quad r=+0.60(p<0.0 \mathrm{I})$

- Poorly controlled diabetics $\quad n=19 \quad r=+0.50(0.02<p<0.05)$

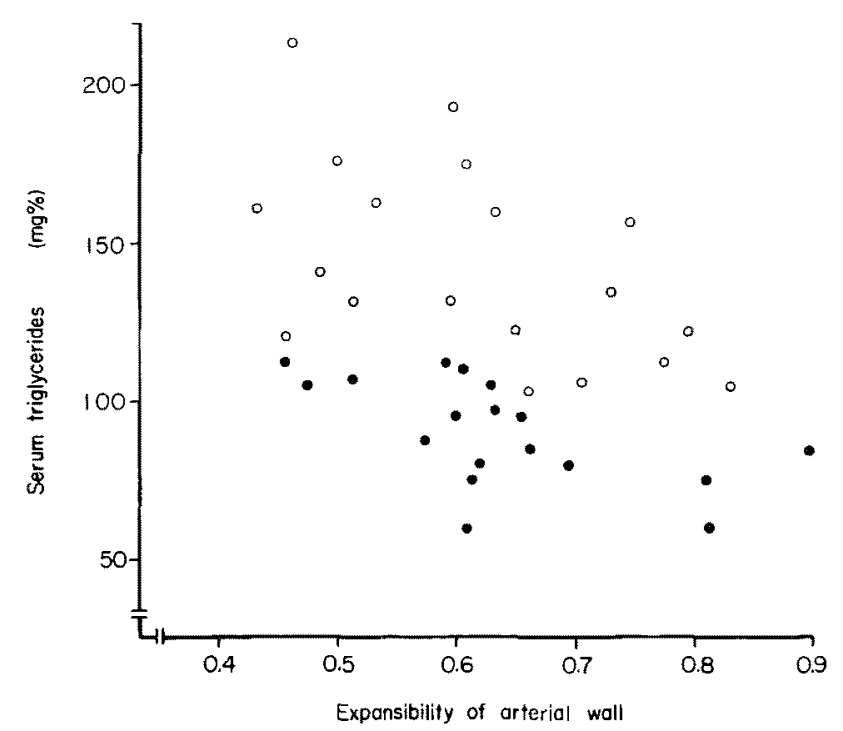

Fig. 4. Relationship between expansibility of arterial wall and serum triglyceride levels in well- and poorly controlled diabeties.

- Well-controlled diabetics $\quad n=18 \quad r=-0.59(0.01<p<0.02)$

- Poorly controlled diabetics $n=19 \quad r=-0.57(0.01<p<0.02)$ 
sclerotic and hypertensive patients. The author found a similar correlation in well-controlled diabetics, but not in poorly controlled ones.

On the other hand, no report on the relationship between the expansibility of arterial wall and serum triglyceride levels has hitherto appeared. In the present study, serum triglycerides were found to have a high degree of negative correlation in both well- and poorly controlled diabetics. The conclusion was of course based on a high degree of positive correlation between the pulse wave velocity and the serum triglyceride levels found in these diabetics.

There is ample evidence that arteriosclerosis, especially atherosclerosis, in diabetes mellitus begins early in the course of diabetes and progresses with age. Moses ${ }^{9}$ presented evidence that autopsied diabetics exhibited more pronounced atherosclerosis in the coronary, aortic and iliac vessels than their age-matched controls. Woolam et al. ${ }^{10}$ reported that the pulse wave velocity of the diabetics was significantly higher than that of normal subjects. These facts indicate that diabetes accelerates atherosclerosis of the middle and larger arteries.

The role of a disturbance of lipid metabolism in the pathogenesis of atherosclerosis has been increasingly acknowledged. In this regard, interest has been focused mainly on cholesterol for many years, but recently the possible role of triglycerides has been noticed since the work of Albrink and his collaborators ${ }^{11,12}$ pointed out that serum triglycerides provided a better separation of diabetic patients with coronary artery disease from normal subjects than did the other lipids.

Therefore, it is of great interest that a close relationship was found between serum triglycerides as well as serum cholesterol and the expansibility of arterial wall that represented quantitatively the degree of arteriosclerosis from the ascending aorta to the radial artery where atherosclerosis occurs frequently.

It is generally accepted that antidiabetic treatments in long duration lower serum triglycerides. ${ }^{13,14}$ However, elevation of serum triglycerides in close association with the decrease in the expansibility of arterial wall even in wellcontrolled diabetics discloses the necessity for a more effective treatment or prevention of arteriosclerosis.

Finally, the measurement of the expansibility seems to be well applicable for reliable detection of arteriosclerosis in the middle and larger arteries.

\section{Summary}

The expansibility of arterial wall in diabetics treated over one year was measured by determining the pulse wave velocity from the origin of the aorta to the radial artery at the wrist and evaluated in relation to the fasting serum triglyceride levels. A good negative correlation between the expansibility of arterial wall and the serum triglyceride levels was found in both well- and poorly controlled. diabetics. A similar correlation was also found with the total serum cholesterol levels only in well-controlled diabetics. These findings indicate a close relationship 
of serum triglycerides and serum cholesterol to the degree of arteriosclerosis in the examined arterial region.

\section{Acknowledgment}

I wish to express my gratitude to Prof. Takeshi Kimura and Assist. Prof. Masataka Kato for their suggestions.

\section{References}

1) Bramwell, J.C. \& Hill, A.V. Velocity of transmission of the pulse-wave. Lancet, 1922, 202, 891-892.

2) Bramwell, J.C. \& Hill, A.V. The velocity of the pulse wave in man. Proc. roy. Soc. $B, 1922,93,298-306$.

3) Kato, M., Sato, T. \& Shioji, R. Study on relationship between blood pressure and pulse wave velocity. Kokyn to Junkan (Jap.), 1960, 8, 379-385.

4) Kato, M. The pulse wave velocity. Igaku no Ayumi (Jap.), 1960, 35, 51-58.

5) Kato, M., Takahashi, M., Takahashi, H., Suzuki, H. \& Shiji, R. Relationship between expansibility of arterial wall and various factors influencing on arteriosclerosis. Rinsho Naika Shonika (Jap.), 1962, 17, 661-665.

6) Zuckerman, J.L. \& Natelson, S. A convenient and rapid procedure for total cholesterol estimation using an acid chloroform extraction. J. Lab. clin. Med., 1948, $33,1322-1325$.

7) Ota, M. An epidemiologic study of serum cholesterol levels. Jap. Circulat.J., 1960, 24, 836-853.

8) Van Handel, E. \& Zilversmit, D.B. Micromethod for the direct determination of serum triglycerides. J. Lab. clin. Med., 1957, 50, 152-157.

9) Moses, C. Distribution and severity of atheroselerotic lesions. In: Atherosclerosis. Mechanisms as a guide to prevention. Edited by C. Moses, Lea \& Febiger, Philadelphia, 1963, p. 88.

10) Woolam, G.L., Schnur, P.L., Vallbona, C. \& Hoff, H.E. The pulse wave velocity as an early indicator of atherosclerosis in diabetic subjects. Circulation, 1962, 25, $533-539$.

11) Albrink, M.J. \& Man, E.B. Serum triglycerides in coronary artery disease. Arch. in. tern. Med., 1959, 103, 4-8.

12) Albrink, M.J., Meigs, J.W. \& Man, E.B. Serum lipids, hypertension and coronary artery disease. Amer. $J$. Med., 1961, 31, 4-23.

13) Adlersberg, D. \& Eisler, L. Circulating lipids in diabetes mellitus. I. Amer. med. Ass., 1959, 170, 1261-1265.

14) Hirata, Y., Omura, K. \& Fujimi, S. Clinical aspects of lipid metabolism in diabetes mellitus, Rinkho Byori (Jap.), 1965, 13, 492-496. 